\title{
Transfer of discriminative control during stimulus fading conducted without reinforcement
}

\author{
Lanny Fields ${ }^{1}$
}

Published online: 8 August 2017

(C) Psychonomic Society, Inc. 2017

\begin{abstract}
Using trial-and-error training, eight pigeons did not learn to discriminate between $45^{\circ}$ and $135^{\circ}$ lines, but did learn to discriminate between red and green colors. Control by line tilt was induced by stimulus fading that did not include reinforcement while fading out the colors. After establishing the red-green discrimination, low-intensity lines were superimposed on colors and were gradually faded in. All of this was done using reinforcement. At the end of the line fade-in, the lines had not acquired control of responding. Finally, color intensity was gradually faded out in the absence of reinforcement, and the lines acquired discriminative control by six of the eight pigeons. Thus, reinforcement during the color fade-out was not necessary for the acquisition of discriminative control by the lines during fading. Acquisition of control by lines was attributed to overshadowing, the reduction of stimulus blocking by generalization, and the evocation of correct responding by the colors while the participants were attending to the lines. This last process was also responsible for the induction of discriminative control during sensory preconditioning, higher order conditioning, and response transfer in equivalence classes. Errors, however, were not correlated with discrimination learning during stimulus fading. Finally, transfer of control occurred very quickly with or without errors.
\end{abstract}

Keywords Stimulus fading · No reinforcement · Errorless learning $\cdot$ Overshadowing $\cdot$ Blocking $\cdot$ SR contiguity

Lanny Fields

lanny.fields1@gmail.com

1 Queens College and The Graduate School of the City University of New York, Queens, NY, USA
Discriminative control of operant behavior is usually established by the differential reinforcement of the same operant in the presence of at least two stimuli (Catania, 2013; Keller \& Schoenfeld, 1950). Specifically, a response is reinforced in the presence of one stimulus called an $\mathrm{S}^{\mathrm{D}}$ and is not in the presence of at least one other called an $\mathrm{S}^{\Delta}$. Continued exposure to these operations increases and decreases responding to the $\mathrm{S}^{\mathrm{D}}$ and $\mathrm{S}^{\Delta}$, respectively, which documents the acquisition of discriminative control of the response. Thus, the formation of the discrimination is attributed to the contingencies of reinforcement. Because many errors occur during the process, the procedure has been said to produce "trial and error learning" (Morgan, 1920).

In some cases, the contingencies of reinforcement do not establish discriminative control by particular stimuli. Indeed, these failures define problematic discriminations. Some examples include responding differentially to lines of different orientations by pigeons (Terrace, 1963), responding differentially to circles and ellipses by people with an intellectual disabilities (Sidman \& Stoddard, 1967; Stoddard \& Sidman, 1967), complying with instructions or requests by oppositional children (Ducharme, Atkinson, \& Poulton, 2000), engaging in activities of daily living by individuals with dementia (De Werde, Boelen, Rikkert, \& Kessels, 2013), remembering by individuals with acquired memory deficits (Clare \& Jones, 2008; Evans et al., 2000), assembling machines such a lawn mower engines by individuals with intellectual disabilities (Walls, Haught, \& Dowler, 1982), and learning the content in some college courses (Heckler, Fuqua, \& Pennypacker, 1975).

This deficit in the efficacy of trial-and-error training was addressed by the use of an alternative paradigm, generically called stimulus fading (Clare \& Jones, 2008; Etzel \& LaBlanc, 1979; Fields, Bruno, \& Keller, 1976; Moore \& Goldiamond, 1964; Schusterman, 1967; Terrace, 1963; Touchette, 1971), which has resulted in the learning of 
problematic discriminations like those mentioned above. Since that learning frequently occurred with few if any errors, stimulus fading has been said to produce "errorless" learning (Rilling, 1977; Terrace, 1963). Indeed, Skinner (1968) proposed that errorless learning should be incorporated extensively into many forms of educational technology. By implication, learning should also occur more quickly by stimulus fading than by trial-and-error training.

Although stimulus fading has many procedural variants (Brown, \& Rilling, 1975; Etzel \& LaBlanc, 1979; Schusterman, 1967; Touchette, 1971; Touchette \& Howard, 1984; Walls et al., 1982; Zygmont, Lazar, Dube, \& Mcllvane, 1992), most of them share the following procedural operations. After demonstrating that trial-and-error training does not result in the acquisition of stimulus control by some response by some target stimuli, that response is brought under the control of a second set of easily discriminable cues, called original controlling stimuli. Then, the salience of each target stimulus is reduced to a low level, and each is superimposed on one of the originals, forming compound stimuli. In the subsequent "fading-in" phase, the intensity of the original controlling stimuli remains high while the salience of each target stimulus is gradually increased as long as the compounds maintain discriminative control. In the penultimate "fading-out" phase, the salience of the originals is gradually attenuated over a series of trials while the salience of the target elements remains high. Finally, the salient target stimuli are presented alone and typically produce differential responding, which documents the acquisition of control by the target stimuli, or the transfer of control from the original stimuli to the new target stimuli.

To my knowledge, all fading procedures have been conducted with contingent reinforcement. All fading procedures, however, also involve the pairing of original and target stimuli while fading in the target stimuli and fading out the original stimuli. Thus, the acquisition of stimulus control by target stimuli could be driven by reinforcement, stimulus pairing, and stimulus-response contiguity (Hall, 1980). The focus the present experiment is to determine whether reinforcement is responsible for the acquisition of discriminative control by the target stimuli during fading.

In virtually all fading studies, the target stimuli acquire stimulus control while fading out the original controlling stimuli. Thus, it would be most likely to show the effects of reinforcement on the acquisition of discriminative control by the target stimuli while fading out the original stimuli (Doran \& Holland, 1979; Fields et al., 1976; Touchette, 1971; but see Fields, 1979) for acquisition during the fading in of the new stimuli. In the present experiment, reinforcement was used while establishing stimulus control by original stimuli (red and green colors) and also during the subsequent superimposition and fading in of target stimuli $\left(45^{\circ}\right.$ and $135^{\circ}$ white lines) on the colors. Thereafter, compound color-line stimuli were presented without reinforcement, and the salience of the colors was gradually attenuated, with line probes presented after each attenuation of color intensity. The emergence of stimulus control by the lines would indicate that reinforcement was not needed for acquisition and could be accounted for by the stimulus-stimulus pairing operations and stimulus-response contiguity embedded in the fading procedure.

\section{Method}

\section{Subjects}

Eight naïve White Carneaux pigeons were maintained at $80 \%$ of their free-feeding weights for the duration of the experiment and were allowed free access to water and grit in their home cages.

\section{Apparatus}

The experiment was conducted in Scientific Prototype threekey operant chambers. Each response key was $25 \mathrm{~mm}$ in diameter. Responses were defined as the depression of a key with a force of at least $0.2 \mathrm{~N}$. Reinforcers consisted of 2.5 -s access to mixed grain. All discriminative stimuli were generated from IEE In-line projectors, the lights in which transilluminated the response keys with red, green, or white fields, or white lines with $45^{\circ}$ or $135^{\circ}$ angular orientations that appeared on dark backgrounds. When the lines were presented alone as probes, they were projected on dark backgrounds. On other trials, the $45^{\circ}$ white line appeared on a red background and the $135^{\circ}$ line appeared on a green background.

The intensities of the red and green colors and the lines were controlled by the values of precision resistors wired in series with the projector bulbs in the IEE projectors (Fields, 1978, 1979; Fields et al., 1976; Karpicke \& Hearst, 1975; Terrace, 1963). Stimulus intensity was varied in a stepwise manner by changing resistance values. Thus, 0 -ohms corresponded to full-intensity stimuli and lower intensity stimuli were correlated with higher values of electrical resistance. Intensity was varied in 2-ohm steps from zero to $10 \mathrm{ohms}$, and in 1-ohm steps from $10 \mathrm{ohms}$ to $24 \mathrm{ohms}$. Changes in stimulus intensity were controlled by use an electromechanical bidirectional stepper, the contacts of which were wired to the various resistors and connected in series to the bulbs that generated the colors and lines. All stimulus presentations and associated reinforcement contingencies were programmed in SuperSKED (State Systems, Inc.) and implemented using a Digital Equipment PDP 8-A minicomputer.

\section{Procedure}

The experiment was conducted in six consecutive phases, each described below. 
Phase 1: Auto-shaping. Phase 1 trained the subjects to respond to red, green, and white stimuli by use of a threekey auto-shaping procedure (P. L. Brown \& Jenkins, 1968). On a given trial, a red, white, or green stimulus was presented on either the left, center, or right key in a block randomized sequence. If the subject did not peck the lighted key, the stimulus presentation lasted $8 \mathrm{~s}$. A single key peck terminated the stimulus presentation. Regardless of responding, immediate access to mixed grain was provided for $2.5 \mathrm{~s}$. Stimulus-food presentations were programmed on a Variable-Time 45-s schedule (VT$45 \mathrm{~s}$ ). This unpredictable time duration that separated the onset of a trial and the presentation of the reinforcer engendered higher and more constant response rates than a fixed time auto-shaping procedure. Each of the three colors was paired with food 15 times in a session, producing sessions containing 45 stimulus-food pairings. Acquisition occurred when correct responses occurred on at least 43 of the 45 trials in two consecutive sessions. This was reached in three to five sessions by all birds.

\section{Phase 2: Training separate color-position response} repertoires. After auto-shaping, two separate colorpositional response repertoires were established using a time-based and then intensity-based fading routine. To establish stimulus control over the left-key response by two red keys, a full-intensity red stimulus ( 0 ohms) was presented on the left key at the same time that a low intensity (16 ohms) red stimulus was presented on the right key. The stimulus on the left key remained on for a maximum duration of $8 \mathrm{~s}$ in the absence of responding, while the low intensity stimulus remained on for $0.25 \mathrm{~s}$. The occurrence of a left-key response terminated both stimuli if they were on and produced $2.5 \mathrm{~s}$ access to grain, after which a VT 45-s intertrial interval (ITI) ensued. Finally, each left-key response emitted in the presence of the red stimulus lengthened the duration of the subsequent right-key stimulus presentation by $0.25 \mathrm{~s}$ until both keys remained on for a maximum duration of $8 \mathrm{~s}$ in the absence of responding. Failure to respond and responding to the right key turned off all stimuli for the ITI, but neither response was followed by food presentation. Also, the duration of the subsequent right key stimulus was reduced by $0.25 \mathrm{~s}$.

After the right-key stimulus was at its full duration, the intensity of the red light was increased contingent upon left-key responding. A response to the left key was reinforced as above and also resulted in a one-step decrease in the resistance value associated with the right-key stimulus for the next trial. A response to the right-key stimulus increased resistance by one step and was not followed by food. The session ended when a left-key response occurred in the presence of eight consecutive trials with both stimuli present at full intensity.
The procedure for establishing control of the right-key response by two green stimuli was similar with the exception that green stimuli were presented on both keys. The terminal performances documented the establishment of a green-left response repertoire.

Phase 3: Simultaneous red-green discrimination. Phase 3 involved the establishment of a simultaneous red-green discrimination in which red and green stimuli were used as the original $\mathrm{S}^{\mathrm{D}} \mathrm{s}$. In each trial, the $\mathrm{S}^{\mathrm{D}} \mathrm{s}$ were changed from two full-intensity red stimuli to two fullintensity green stimuli in random order. Reinforcement was provided for pressing the left key in the presence of the two red stimuli, and pressing the right key in the presence of two green stimuli. Each trial began by illuminating the center key with a full-intensity white light that remained on until pecked by a subject. As the white stimulus terminated, both side keys were transilluminated with full-intensity red or green lights for up to $8 \mathrm{~s}$. The first response to either side key turned off the lights for a 10-s intertrial interval (ITI). The stimuli were also turned off for the same ITI if neither side key was pecked within $8 \mathrm{~s}$ of stimulus onset. The sequence of red and green trials was block randomized so that an equal number of red and green trials occurred in a session, each of which contained 50 trials. Phase 3 lasted up to four sessions, and ended when a subject responded accurately on at least $95 \%$ of trials in a session. Accurate responding then demonstrated that the procedure prevented the development of a position bias and also prevented the pecking the stimulus immediately in front of the pigeon.

Phase 4: Fading in lines. In the next session, the first 20 trials continued the training of the previously established red-green discrimination. Beginning with the 21 st trial, red or green stimuli were no longer presented. Instead, each trial involved the transillumination of both side keys with either full-intensity $45^{\circ}$ lines or $135^{\circ}$ lines, all of which were presented on dark backgrounds. Called probes, these trials were administered under extinction conditions until eight consecutive trials terminated without responding, a criterion that was met in 10-17 trials. These performances then showed that the two lines were not discriminative for positional responding.

The session then continued with trials that contained full-intensity colors and lines, called compound stimuli. Initially, low-intensity lines (18 ohms) were superimposed on the colors forming compound bright red-dim $45^{\circ}$ and bright green-dim $135^{\circ}$ stimuli. Responding on the left key was reinforced in the presence of the red $-45^{\circ}$ compound, and responding on the right key was reinforced in the presence of the green- $135^{\circ}$ compound.

Line intensity increased in 1-ohm steps every time two consecutive trials were terminated by correct responses. In contrast, line intensity decreased by a 1-ohm step each 
time a trial was terminated by an incorrect response (pressing the wrong key or not pressing either key within $8 \mathrm{~s}$ of trial onset). Once line stimuli reached full intensity ( 0 ohms), all subsequent trials contained full-intensity lines and colors. Training continued with full-intensity compounds until eight consecutive trials produced correct responses. Once achieved, control by lines was assessed by presenting one $45^{\circ}$ and one $135^{\circ}$ line probe under extinction conditions.

Phase 5: Maintenance of the compound color-line discrimination. In the final phase of the experiment (Phase 6), colors were faded out under extinction conditions. To increase the likelihood that responding would persist while the colors were being faded out, Phase 5 was conducted to maintain correct responding in the context of increasingly intermittent reinforcement. Throughout Phase 5, all trials involved the presentation of compound stimuli that contained full-intensity colors and lines. For three sessions, correct responding was always reinforced. Thereafter, the probability of reinforcing correct responses was reduced in every other session to $0.5,0.40$, 0.30 , and 0.25 , successively. Finally, responding was reinforced with a probability of 0.20 for five sessions. The last such session ended with the presentation of one $45^{\circ}$ and one $135^{\circ}$ probe trial under extinction conditions to determine whether the lines had acquired stimulus control while reducing reinforcement density.

Phase 6: Fading out of colors in extinction. In this final phase, colors were faded out under extinction conditions. Each compound stimulus contained full-intensity lines, with colors presented at increasingly lower intensities. As colors were faded out, line probe stimuli were presented periodically to monitor the emergence of discriminative control acquired by the lines (Doran \& Holland, 1979; Fields, 1978, 1979, 1980, 1981, 1985; Fields, et al., 1976).

Fading out of the colors began with the presentation of a block of four compounds, each of which contained fullintensity lines and colors. If correct responses are made to all four compounds, a pair of probe trials was presented (one trial with a $45^{\circ}$ line and another with a $135^{\circ}$ line). Regardless of responding to the probe trials, the next block of compound stimuli was presented with the colors at a lower intensity and the lines at full intensity.

If a trial in the first block of four was terminated by an incorrect response, that event cancelled the accumulation of correct trials and scheduled the presentation of up to four more compound trials at the same fading level. Correct responding to the next four compounds resulted in the presentation of two line probes and a reduction in the intensity of color used in the next four compounds. Responding to the line probes tracked the emergence of discriminative control acquired by the lines.
On the other hand, if a second compound trial produced an incorrect response, the next trial initiated a new block of compounds with the color intensity one step brighter than on the preceding trial; in addition, no probes were presented prior to this increase in color salience.

Acquisition of control by lines was demonstrated when a subject responded correctly to eight consecutive probe stimuli presented after four consecutively presented fading levels. Because chance likelihood of occurrence was very low: $.5^{8}$ or $p<.004$, these probe performances provided a strong measure of the acquisition of dimensional control by the lines. The line fade-out session terminated after demonstrating acquisition, after 400 trials without achieving acquisition, or when responding stopped before the 400th trial.

\section{Results}

\section{Performances in Phases 1 through 5}

After successful auto-shaping in Phase 1, all of the subjects acquired the red-green discrimination in Phases 2 and 3. At the start of Phase 4, the lines did not produce responding and were then superimposed and faded-in on the colors. During the line fade-in, discriminative control by colors was maintained by all subjects. In the subsequently presented line probe set, neither probe evoked a response, indicating that the lines had not acquired discriminative control while being faded in for any subject. For all subjects in Phase 5, the compounds continued to exert discriminative control during the feedback reduction while the line probes at the end of the phase still did not produce responses. Thus, feedback reduction did not induce control by line tilt.

\section{Performances in Phase 6: color fade-out}

The color fade-out phase of the experiment involved the presentation blocks of compound stimuli and blocks of line probes. The compound stimuli in a block and the subsequently presented block of line probes could produce three patterns of responding that were of interest. First, a submastery level of responding produced by the compounds would indicate the absence or the loss of control by the colors. Second, mastery levels of responding produced by the compounds, but not by the probes, would demonstrate control of responding by the colors and a lack of control by the lines. Third, mastery levels of responding produced by the compounds and by the probes would indicate control by colors and by line orientation. Figure 1 shows the performances produced by the compounds and probes during the color fade-out for each subject. 
Errorless Transfer

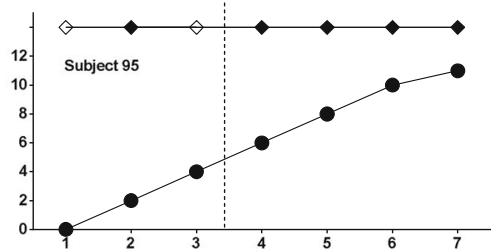

Errorful Transfer

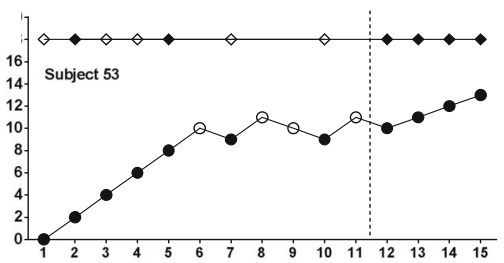

Failed Transfer

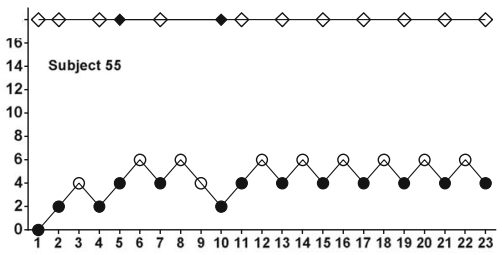

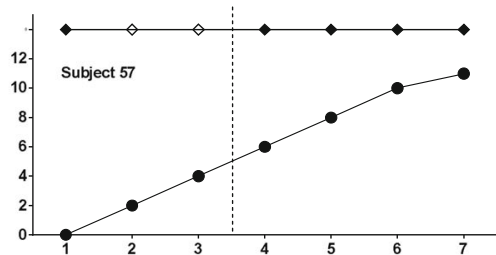
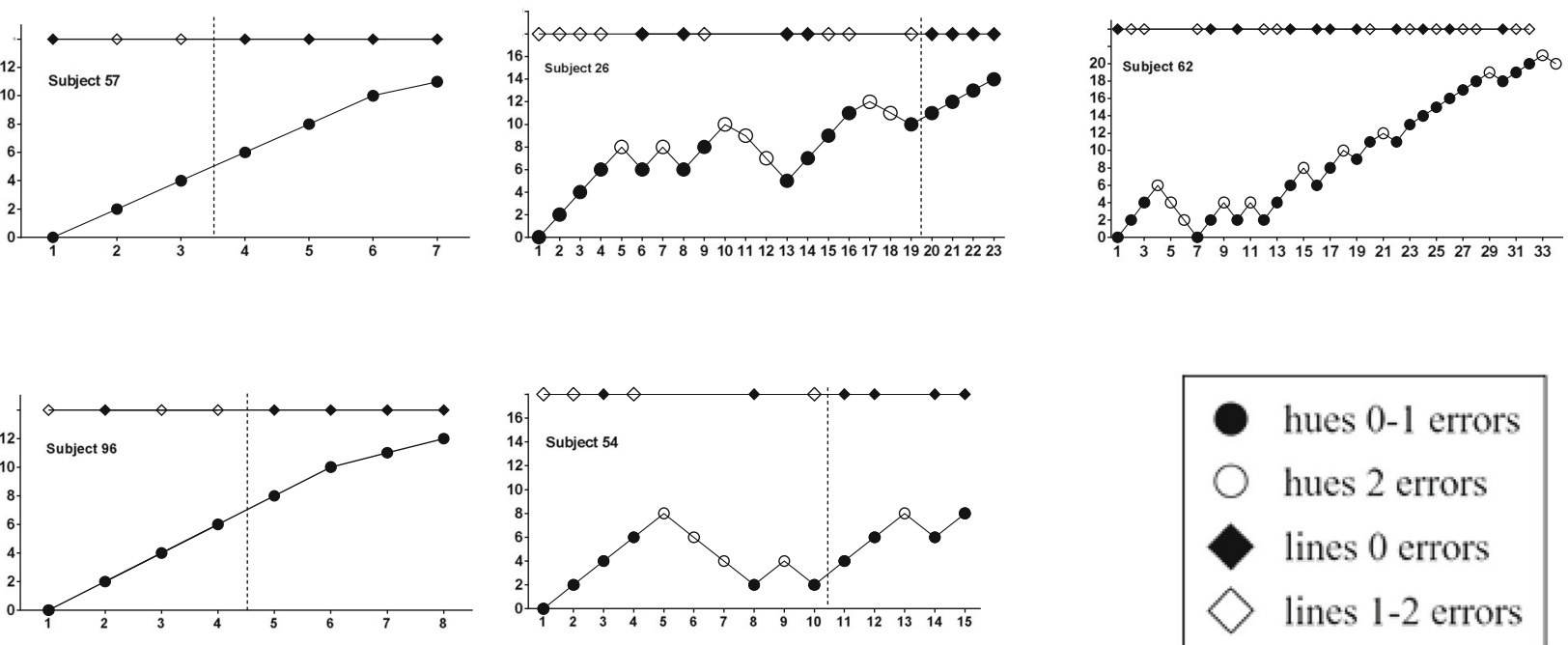

\section{Successive Fading Levels}

Fig. 1 Each panel shows a different subject. In each panel, the lower function depicts the intensity of the colors at each fading level plotted as a function of order of administering the fading levels. Black and white circles on this function indicate maintenance and loss of stimulus control by the compounds, respectively. The upper function indicates

Errorless transfer The panels in the leftmost column depict the results for Subjects 95, 57, and 96, each of whom showed nearly errorless acquisition of stimulus control in the absence of reinforcement during color fade-out. For each subject, the compound stimuli at every fading level produced mastery levels of responding. Thus, each fading level was followed by a reduction in color intensity with no backups. Momentary control by line tilt was documented by the probe data indicated by the upper function in each panel. For these subjects, the lines did not produce responding indicative of control in the initial probe blocks even though the corresponding compound blocks indicated maintained control by the colors. Thereafter, the last four probe blocks documented control by the line tilts, and thus the transfer of discriminative control from colors to lines. On average, $7.3 \mathrm{fad}-$ ing levels were presented to these subjects. Errors were produced by zero of $28(0 \%)$, one of $32(3 \%)$, and two of $36(5 \%)$ of the compound trials for Subjects 95, 96, and 57, respectively. Thus, a line tilt discrimination formed with little to no errors while fading out colors in absence of reinforcement. performances produced by the probes, with black and white diamonds indicating the presence or absence of control by lines alone, respectively. Data for the probe block that follows a given compound block are shown in the same vertical plane

Errorful transfer The panels in the middle column depict the results for Subjects 53, 26, and 54, each of whom also showed the acquisition of stimulus control by line tilt in the absence of reinforcement, albeit with the occurrence of some backups and many more errors than those produced by the three subjects in the leftmost column. At some fading levels, the compounds produced the mastery levels of responding and was followed by a reduction in the intensity of the colors in the subsequent fading level. At other fading levels, the compounds produced two errors that indicated a loss of control by the current color intensity and resulted in an increase in color intensity in the next block of compound stimuli. When the probe performances were considered, some probe pairs produced two correct responses, while others did not. The last four such blocks produced accurate responding that documented the acquisition of stimulus control by line tilt.

When corresponding compound and probe performances were compared, sometimes, the compounds at one fading level demonstrated control by the color while the probe 
performances did not indicate control by the line tilts (a black circle and a white diamond) and sometimes control was exerted by colors and lines (a black circle and a black diamond), but neither of these patterns occurred across adjacent fading levels. Thus, control by the compounds at one fading level did not necessarily predict the presence of stimulus control by the lines. Eventually, however, control of responding was shown on a continuing basis by the colors and lines across the last four fading levels (black circles and black diamonds). On average, 19 fading levels were needed for these subjects to acquire the line tilt discriminations. Errors were produced by 21 of $86(24 \%), 24$ of $96(23 \%)$, and 13 of $61(21 \%)$ of the compounds for subjects 53,26 , and 54 , respectively.

Failed transfer The panels in right most column depict the results for subjects 55 and 62 , both of whom failed to acquire the line tilt discrimination. Subject 55 oscillated between fading Levels 2 and 4, failing to master the compound discrimination at Level 4, reverting to fading Level 2, at which point the subject immediately remastered control by the colors. In 11 of the 13 probe sets, the line probes did not exert stimulus control even though the colors did so at that fading level. Thus, two closely separated color intensities (e.g., two and four) did and did not exert control of responding, respectively, while the lines never acquired control of responding.

For Subject 62, the first 13 steps of color fade-out were characterized by decreases and increases in fading levels and reflected variation in control by color across successive fading levels. The subsequent steps involved systematic decreases in color intensity across fading levels, which reflected maintained control by increasingly lower intensities of the colors. When the probes were considered, however, the lines showed oscillation between responding correctly and incorrectly across test blocks that probably reflected random responding to the lines probes.

Time to complete color fade-out The prior forms of analysis used accuracy of responding as the primary measure to track stimulus control transfer. Another global measure-time to complete the color fade-out - can also be used to characterize the performances observed in the experiment. When the duration of color fade-out was considered, transfer occurred errorlessly in an average of 13 minutes, and with errors in an average of 29.6 minutes. When transfer did not occur, the color fade phase lasted for an average of 39.8 minutes. The completion times were significantly different across these subgroups, ANOVA, $F(2,5)=17.62, p=.0055$. Dunnett's tests confirmed that errorless transfer occurred significantly faster than errorful transfer, $q(5)=4.039$, and failed transfer, $q(5)=$ 5.695 .

\section{Discussion}

\section{Main findings and effects of reinforcement}

The experiment began with the use of contingent reinforcement to establish a discrimination between red and green colors, which were the original controlling stimuli. Thereafter, white $45^{\circ}$ and $135^{\circ}$ lines were superimposed on the colors, forming compound red- $45^{\circ}$ and green- $135^{\circ}$ stimuli, and then were gradually increased in intensity, all in the context of differential reinforcement. Although correct responding was maintained throughout the process, the superimposition and fading in of lines on colors did not induce discriminative control by line orientation. In the next phase, the full intensity color-line compounds were presented with increasingly diminished likelihoods of reinforcement, and the line probes still did not indicate the acquisition of discriminative control. Therefore, reinforcement during line fade in and during reinforcement reduction did not induce control of behavior by the new line stimuli.

In the final phase, the intensities of the colors in the compounds were gradually faded out, and of most import, in absence of reinforcement. Under those conditions, six of the eight subjects showed the acquisition of stimulus control by line tilt. Therefore, the reinforcement of responding while fading out of the colors was not needed for the lines to acquire discriminative control of responding. In addition, it would appear that the primary role played by differential reinforcement was in the establishment of the original stimulus setthe colors.

Of the six subjects who acquired the line tilt discrimination, three made zero to two errors and three others made 13 to 24 errors in the compound trials administered during color fadeout. Thus, the prevalence of errors (given this range of values) did not influence the likelihood of acquiring the line tilt discrimination in the absence of reinforcement. Terrace (1963) reported that the pigeons in his experiment made fewer than 25 errors during fading while learning new discriminations and characterized these performance as showing "errorless" learning. By that criterion, all six pigeons in the present experiment showed errorless learning, but in the absence of reinforcement, although the first three could be viewed as having learned the discriminations in a virtually errorless manner.

Although reinforcement was not necessary for the induction of stimulus control during the attenuation of the colors, it still could have influenced the likelihood of learning the new line tilt discrimination. In the vast majority of stimulus fading studies, reinforcement was used throughout the procedure and virtually all of the subjects acquired the new discriminations. By contrast, $75 \%$ (six of eight) subjects in the present experiment acquired discriminative control by new stimuli while fading out the originals in the absence of reinforcement. At most, then, the inclusion of reinforcement could have 
increased likelihood of stimulus control acquisition by $25 \%$ while fading out of the original controlling stimuli. Thus, reinforcement while fading out the original controlling stimuli could have provided a small increment in the likelihood of learning the new discriminations. It might also reduce the prevalence of errors during fading.

As already noted, the present experiment demonstrated that stimulus control transfer can occur in the absence of reinforcement during the fading out of the original controlling stimuli. This finding was observed in the context of a specific trial format. To what extent, then, would the nonreinforcement effect also be observed in the context of other successive discrimination formats in which stimuli are presented at one location that is separated from the regions used to define responding, simultaneous discrimination, and conditional discrimination training procedures? Transfer of stimulus control in each of these contexts would attest to the generality of the finding reported in the present experiment.

\section{Acquisition of discriminative control by associative SS and SR processes}

If differential reinforcement does not account for the acquisition of stimulus control during fading, what operations could account for such an outcome? The following discussion considers how stimulus-stimulus associations (SS) and stimulusresponse contiguity (SR) could account for the lack of stimulus control acquisition during the fading in of new stimuli and the acquisition of discriminative control while fading out of the original controlling stimuli.

Lack of acquisition during the fade-in At the start of the line fade-in, the full-intensity lines did not exert discriminative control of responding. After being faded in on the colors, the full-intensity lines still did not exert discriminative control. The absence of control by lines probably reflected the effects of blocking (Kamin, 1968) and overshadowing (Mackintosh, 1976).

Blocking is a phenomenon in which the existence of discriminative control exerted by one set of stimuli interferes with the acquisition of control by a new stimuli that are subsequently superimposed on the first. Stimulus overshadowing is a phenomenon in which the presence of a salient stimulus interferes with attention paid to another concurrently presented stimulus that has lesser salience. Rescorla (1988) noted that blocking is driven by the predictability of reinforcement by the stimuli used in an experimental preparation. By the time pigeons reached Phase 4 of training, they had been exposed to at least 300 trials in which food was available in the presence of hues, while food was never available after the presentation of the lines. Because colors and lines were perfect predictors of reinforcement before pairing and the lines were redundant with the colors, the colors block the acquisition of control by the lines (Pearce, Dopson, Haselgrove, \& Esber, 2012). Because these processes characterize the relations between the colors and the lines as the lines were being faded in, blocking and overshadowing were the probable causes of the absence of discriminative control acquired by the lines in that phase of the experiment.

Acquisition of control during color fade-out The last phase of the experiment involved the gradual attenuation of color intensity while holding constant the high intensities of the lines, all in the absence of reinforcement. The acquisition of control by the lines can be accounted for by considering how attention shifts from colors to lines by the effects of overshadowing, reducing the effects of blocking by stimulus generalization, and the induction of control by lines through stimulus-response contiguity (Guthrie, 1959).

Maintenance of responding. For stimulus control transfer to occur in the absence of reinforcement, the colors had to exert discriminative control as they were being faded out (Fields, 1978; Fields et al., 1976). This was accomplished by the increasingly intermittent reinforcement of the red- $45^{\circ}$ green- $135^{\circ}$ discrimination prior to the color fade-out. The efficacy of this strategy was confirmed by the maintenance of discriminative control by the compounds throughout the color fade-out phase of the experiment.

Attention to lines while fading out of colors. At the start of the color fade-out, the full-intensity colors blocked the acquisition of control by the lines. Thereafter, as the intensities of the colors were systematically diminished, the subjects began to attend to the lines (Fields, 1978). Such an attentional shift was probably driven by overshadowing and blocking effects. With regard to overshadowing, as the colors were being diminished in intensity, the concurrently presented full-intensity lines, of necessity, became more salient. Thus, the overshadowing of lines by colors was followed by the overshadowing of colors by lines.

The effect of blocking during the color fade-out can be understood by considering stimulus generalization. By definition, after a discrimination has been established with one stimulus (e.g., 0 -ohm colors), the presentation of other stimuli that are increasingly distant from the training stimulus along some dimension (e.g., 2-, 6-,...10-ohm colors) should produce responding that is an inverse function of the separation of the latter from the former. This phenomenon is called stimulus generalization. Although it has not yet been measured, it is plausible to assume that the magnitude of stimulus blocking should also decline with increases in the disparity that separates the attenuated colors from the full intensity colors used in red-green discrimination training. Thus, diminishing the blocking effect of the colors while fading out the colors should 
increase the likelihood of attending to the concurrently presented lines.

The argument just presented is predicated on the assumption that the magnitude of stimulus blocking is malleable, as demonstrated by Blaisdell, Gunther, and Miller (1999). Once independently confirmed, generalization would be another variable that would document the malleability of stimulus blocking.

Acquisition during color fade-out. Logically, for the lines to acquire dimensional stimulus control, the subject must be attending to the lines and the colors and must also be responding accurately to the red and green stimuli. Guthrie (1959) proposed that new stimulus-response relations can be induced by SS and SR contiguity. Specifically, a new stimulus would acquire control of a particular response if the new stimulus was being attended to at the same time that a given response was being evoked by another stimulus, all in the absence of reinforcement. Hall (1980) also noted that the formation of new discriminations was enhanced by prior "exposure" or "perceptual" learning, which refer to the same set of operations noted by Guthrie.

These conditions were satisfied in the present experiment. First, the desired responses were evoked by the original red and green elements of the compound stimuli. Second, the attenuation of the colors induced attention to the lines, as described above. Third, even though the salience of the colors was decreasing, they still evoked appropriate positional responding. Fourth, since the subject was attending to the new line stimuli and an appropriate response has been produced, by contiguity, a linkage was established between the line stimuli and the appropriate response, which resulted in the emergence of the $45^{\circ}$-left- $135^{\circ}$-right discrimination. Thus, contemporaneous reinforcement was not needed to account for the acquisition of discriminative control while fading out the original controlling stimuli.

Generality of processes The prior section posited an account of stimulus control acquisition that was produced by SR contiguity: specifically attending to new stimuli while initial stimuli evoked responses that then came under the control of the new stimuli, and in the absence of contemporaneous reinforcement. These conditions also prevail in sensory preconditioning (Brogden, 1939; Rescorla, 1980), higher order conditioning (Rizley \& Rescorla, 1972), and the transfer of responding among the members of an equivalence class (Augustson \& Dougher, 1997; Barnes, Browne, Smeets, \& Roche, 1995; Fields, Adams, Buffington, Yang, \& Verhave, 1996; Lovett, Rehfeldt, Garcia, \& Dunning, 2011; Sidman, 1994; Sidman \& Tailby, 1982).

With regard to sensory preconditioning, after the pairing of two neutral stimuli (e.g., NS1 $\rightarrow$ NS2), the subsequent use of one as a conditional stimulus (CS) in a classical conditioning paradigm (e.g., NS2 $\rightarrow$ US) results in the elicitation of the conditional response (CR) by the other neutral stimulus (NS1). With regard to higher order conditioning, when one stimulus (NS1) us used as a CS in a classical conditioning paradigm (e.g., NS1 $\rightarrow$ US), and is subsequently paired with another neutral stimulus (NS2 $\rightarrow \mathrm{NS} 1$ ), the NS2 stimulus comes to function as a CS even though it has not been paired directly with a US. With regard to response transfer in an equivalence class (e.g., A-B-C-D-E), a response trained to occur in the presence of one member of an equivalence class (e.g., $\mathrm{E}: \mathrm{R} \rightarrow \mathrm{S}^{\mathrm{r}}$ ) routinely generalizes or transfers with very high probability to the remaining class members (e.g., A:R, $\mathrm{B}: \mathrm{R}, \mathrm{C}: \mathrm{R}, \mathrm{D}: \mathrm{R}$ ) without direct reinforcement (Augustson \& Dougher, 1997; Barnes et al., 1995; Sidman \& Tailby, 1982). All of these phenomena, then, illustrate how stimulus control acquisition can be driven by the contiguity of stimulus-stimulus and stimulus-response relations, in the absence of contemporaneous reinforcement.

\section{Summary and implications}

New stimuli acquired discriminative control while fading out the original controlling stimuli in the absence of reinforcement. Thus, contemporaneous reinforcement was not necessary for the new stimuli to acquire discriminative control during fading. Acquisition occurred even though some errors were emitted in the process. The primary function served by reinforcement was to establish discriminative control by the initial stimuli, while stimulus pairing operations (overshadowing and blocking), the decremental effect of generalization on blocking, and stimulus-response contiguity accounted for the acquisition of discriminative control by the new stimuli. These procedures produced rapid acquisition of difficult to form discriminations. Thus, rapid discrimination learning does not require the inclusion of a reinforcement contingency during all phases of discrimination training.

Author note My thanks to Stephen Fath for his assistance in the conduct of the experiment and preliminary analyses of the data. Reprints may be obtained from Lanny Fields, P.O. Box 733, South Egremont, MA, 01258 (e-mail:lanny.fields1@gmail.com).

\section{Compliance with ethical standards}

Funding This research was supported by PSC/BHE Faculty research Awards 12232, 13013, and 13490, granted by the City University of New York.

Conflict of interest The authors declare that there is no conflict of interest.

Ethical approval All procedures performed in studies involving animal subjects were in accordance with the ethical standards and with the 1964 Helsinki declaration and its later amendments or comparable ethical standards. 


\section{References}

Augustson, E. M., \& Dougher, M. J. (1997). The transfer of avoidance functions through stimulus equivalence classes. Journal of Behavior Therapy and Experimental Psychiatry, 28, 181-191.

Barnes, D., Browne, M., Smeets, P., \& Roche, B. (1995). A transfer of functions and conditional transfer of functions through equivalence relations in three- to six-year-old children. The Psychological Record, 45, 405-430.

Blaisdell, A. P., Gunther, L. M., \& Miller, R. R. (1999). Recovery from blocking achieved by extinguishing the block CS. Animal Learning and Behavior, 27, 63-76.

Brogden, W. J. (1939). Sensory pre-conditioning. Journal of Experimental Psychology, 25(4), 323-332. doi:10.1037/h0058944

Brown, P. L., \& Jenkins, H. M. (1968). Auto-shaping of the pigeon's key peck. Journal of the Experimental Analysis of Behavior, 11, 1-8.

Brown, C. H., \& Rilling, M. E. (1975). Stimulus delay and the reduction of errors in the transfer of stimulus control. Animal Learning and Behavior, 3, 21-27.

Catania, A. C. (2013). Learning (5th ed.). Cornwall on Hudson, NY: Sloan.

Clare, L., \& Jones, R. (2008). Errorless learning in the rehabilitation of memory impairment: A critical review. Neuropsychological Rehabilitation, 18, 1-23.

De Werde, M. M. E., Boelen, D., Rikkert, M. G. M. O., \& Kessels, R. (2013). Errorless learning of everyday tasks in people with dementia. Clinical Interventions in Aging, 8, 1177-1190.

Doran, J., \& Holland, J. (1979). Control by stimulus features during fading. Journal of the Experimental Analysis of Behavior, 31, $177-188$.

Ducharme, J. M., Atkinson, L., \& Poulton, L. (2000). Success-based, non-coercive treatment of oppositional behavior in children from violent homes. Journal of the American Academy of Child and Adolescent Psychiatry, 39, 995-1004.

Etzel, B. C., \& LaBlanc, J. M. (1979). The simplest treatment alternative: The law of parsimony applied to choosing appropriate instructional control and errorless-learning procedures for the difficult-to-teach child. Journal of Autism and Developmental Disorders, 9, 361-382.

Evans, J. J., Wilson, B. A., Schuri, U., Andrade, J., Baddeley, A., Bruna, O., ... Taussik, I. (2000). A comparison of "errorless" and "trialand-error" learning methods for teaching individuals with acquired memory deficits. Neuropsychological Rehabilitation, 10, 67-101.

Fields, L. (1978). Fading and errorless transfer in successive discriminations. Journal of the Experimental Analysis of Behavior, 30, 123128.

Fields, L. (1979). Acquisition of stimulus control while introducing new stimuli in fading. Journal of the Experimental Analysis of Behavior, 32, 121-127.

Fields, L. (1980). Enhanced learning of new discriminations after stimulus fading. Bulletin of the Psychonomic Society, 15, 327-330.

Fields, L. (1981). Early and late introduction of probes and stimulus control acquisition in fading. Journal of the Experimental Analysis of Behavior, 36, 363-370.

Fields, L. (1985). Reinforcement of probe responses and acquisition of stimulus control in fading procedures. Journal of the Experimental Analysis of Behavior, 36, 363-370.

Fields, L., Adams, B. J., Buffington, D. M., Yang, W., \& Verhave, T. (1996). Response transfer between stimuli in generalized equivalence classes: A model for the establishment of natural kind and fuzzy superordinate categories. The Psychological Record, 46, 655-684.

Fields, L., Bruno, V., \& Keller, K. (1976). The stages of acquisition in stimulus fading. Journal of the Experimental Analysis of Behavior, 26, 295-300.
Guthrie, E. R. (1959). Association by contiguity. In S. Koch (Ed.), Psychology: A study of a science (pp. 158-195). New York, NY: McGraw-Hill.

Hall, G. (1980). Exposure learning in animals. Psychological Bulletin, 88, 535-550.

Heckler, J. B., Fuqua, R. W., \& Pennypacker, H. S. (1975). Errorless differentiation of academic responses by college students. Teaching of Psychology, 2, 103-107.

Kamin, L. J. (1968). "Attention-like" processes in classical conditioning. In M. R. Jones (Ed.), Miami symposium on the prediction of behavior: Aversive stimulation (pp. 9-31). Miami, FL: University of Miami.

Karpicke, J., \& Hearst, E. (1975). Inhibitory control and errorless discrimination learning. Journal of the Experimental Analysis of Behavior, 23, 158-166.

Keller, F. S., \& Schoenfeld, W. N. (1950). The principles of psychology. New York, NY: Appleton-Century-Crofts.

Lovett, S., Rehfeldt, R. A., Garcia, Y., \& Dunning, J. (2011). Comparison of a stimulus equivalence protocol and traditional lecture for teaching single-subject designs. Journal of Applied Behavior Analysis, 44, 819-833.

Mackintosh, N. J. (1976). Overshadowing and stimulus intensity. Animal Learning \& Behavior, 4, 186-192. doi:10.3758/BF03214033

Moore, R., \& Goldiamond, I. (1964). Errorless establishment of visual discrimination using fading procedures. Journal of the Experimental Analysis of Behavior, 7, 269-272.

Morgan, C. L. (1920). Animal behavior. London, UK: Edward Arnold.

Pearce, J. M., Dopson, J. C., Haselgrove, M., \& Esber, G. R. (2012). The fate of redundant cues during blocking and a simple discrimination. Journal of Experimental Psychology: Animal Behavior Processes, 38, 167-179. doi:10.1037/a0027662

Rescorla, R. A. (1980). Simultaneous and successive associations in sensory preconditioning. Journal of Experimental Psychology: Animal Behavior Processes, 6, 207-216. doi:10.1037/0097-7403.6.3.207

Rescorla, R. A. (1988). Pavlovian conditioning. It's not what you think it is. American Psychologist, 43, 151-160.

Rilling, M. (1977). Stimulus control and inhibitory processes. In W. K. Honig \& J. E. R. Staddon (Eds.), Handbook of operant behavior. Prentice Hall: Englewood Cliffs, NJ.

Rizley, R. C., \& Rescorla, R. A. (1972). Associations in second-order conditioning and sensory pre-conditioning. Journal of Comparative and Physiological Psychology, 81, 1-11.

Schusterman, R. J. (1967). Attention shift and errorless reversal learning by the California sea lion. Science, 156, 833-835.

Sidman, M. (1994). Equivalence relations and behavior: A research story. Boston, MA: Authors Cooperative.

Sidman, M., \& Stoddard, L. T. (1967). The effectiveness of fading in programming a simultaneous form discrimination for retarded children. Journal of the Experimental Analysis of Behavior, 10, 3-15.

Sidman, M., \& Tailby, W. (1982). Conditional discrimination vs. matching to sample: An expansion of the testing paradigm. Journal of the Experimental Analysis of Behavior, 37, 5-22.

Skinner, B. F. (1968). The technology of teaching. New York, NY: Appleton-Century-Crofts.

Stoddard, L. T., \& Sidman, M. (1967). The effects of errors on children's performance on a circle-ellipse discrimination. Journal of the Experimental Analysis of Behavior, 10, 261-270.

Terrace, H. S. (1963). Errorless transfer of a discrimination across two continua. Journal of the Experimental Analysis of Behavior, 6, 223 232.

Touchette, P. E. (1971). Transfer of stimulus control: Measuring the moment of transfer. Journal of the Experimental Analysis of Behavior, 15, 347-354.

Touchette, P. E., \& Howard, J. (1984). Errorless learning: Reinforcement contingencies and stimulus control transfer in delayed prompting. Journal of Applied Behavior Analysis, 17, 175-181. 
Walls, R. T., Haught, P., \& Dowler, D. L. (1982). Moments of transfer of stimulus control in practical assembly tasks by mentally retarded adults. American Journal of Mental Deficiency, 87, 309-315.
Zygmont, D. M., Lazar, R. M., Dube, W. V., \& McIlvane, W. J. (1992). Teaching arbitrary matching via sample stimulus-control shaping to young children and mentally retarded individuals: A methodological note. Journal of the Experimental Analysis of Behavior, 57, 109118. 